HIFAN 1773

\title{
Some Considerations Regarding Pulsed Correction of Chromatic Aberrations in Final Focusing Systems by
}

\author{
R.O. Bangerter \\ Lawrence Berkeley National Laboratory (on behalf of U.S. HIFS-VNL) \\ 1 Cyclotron Road, Berkeley, CA 94720
}

Lawrence Berkeley National Laboratory

Accelerator Fusion Research Division

Ernest Orlando Lawrence Berkeley National Laboratory

University of California

Berkeley, California 94720

\section{March 31, 2010}

This document was prepared as an account of work sponsored by the United States

Government. While this document is believed to contain correct information, neither the United States Government nor any agency thereof, nor The Regents of the University of California, nor any of their employees, makes any warranty, express or implied, or assumes any legal responsibility for the accuracy, completeness, or usefulness of any information, apparatus, product, or process disclosed, or represents that its use would not infringe privately owned rights. Reference herein to any specific commercial product, process, or service by its trade name, trademark, manufacturer, or otherwise, does not necessarily constitute or imply its endorsement, recommendation, or favoring by the United States Government or any agency thereof, or The Regents of the University of California. The views and opinions of authors expressed herein do not necessarily state or reflect those of the United States Government or any agency thereof or The Regents of the University of California.

This work was supported by the Director, Office of Science, Office of Fusion Energy Sciences, of the U.S. Department of Energy under Contract No. DE-AC0205CH11231. 


\title{
Some Considerations Regarding Pulsed Correction of Chromatic Aberrations in Final Focusing Systems
}

\author{
Roger Bangerter \\ 31 March 2010
}

Nearly all designs of accelerators for heavy ion fusion rely on a velocity (energy) ramp to compress the beam longitudinally from its length in the accelerator to the length required at the target. The size of the velocity ramp is constrained by the longitudinal emittance of the beam. For example, if the longitudinal emittance is $0.05 \mathrm{eV} \cdot \mathrm{s}$ and we wish to produce a pulse having a width of $\pm 2.5 \mathrm{~ns}$ at the target, we must supply an energy tilt such that the energy spread at the target is at least $\pm 0.05 \mathrm{eV} \bullet \mathrm{s} / 2.5 \mathrm{~ns}= \pm 2 \times 10^{7} \mathrm{eV}$. The minimal value of energy spread occurs when the beam has propagated to the point where there is no correlation between the time and energy variables of the beam particles. (In the simple approximation where the boundary of the longitudinal phase space containing the particles is an ellipse, the ellipse is erect at this point, i.e., not tilted with respect to the axes.) In any case, the energy spread can affect focusing. If, for example, the beam kinetic energy is of the order of $5 \mathrm{GeV}$, a tilt of $\pm 2 \times 10^{7} \mathrm{eV}$ corresponds to a fractional energy spread of 0.004 and it may be possible to focus the beam to the required spot size without using an achromatic optical system. Nevertheless, an optical system that allows larger longitudinal emittance should lead to a less expensive accelerator since the tolerances on acceleration waveforms could be relaxed. Moreover, at lower kinetic energies the problem becomes more serious. If the kinetic energy of our example beam were $1 \mathrm{GeV}$ rather than $5 \mathrm{GeV}$, the fractional energy spread would be 0.02 . This much energy spread would likely produce serious chromatic aberrations leading to an unwanted increase in focal spot size. It is interesting to note that the lower limit on energy spread at the target does not depend on whether the beam is neutralized as it approaches the target. If the beam is not neutralized, it will require a larger initial velocity tilt to overcome longitudinal space-charge forces; but these forces will remove part of the tilt as the beam compresses.

Al Maschke suggested that it is possible to reduce the chromatic aberrations by applying a time-dependent transverse focusing correction to the beam upstream of the final focusing lenses [1]. At this point, because of the energy tilt, there is a correlation between longitudinal position in the beam and particle energy. In other words, the average beam energy at the tail of the beam is larger than the average beam energy at the head of the beam. If the beam is completely neutralized as it drifts toward the final focusing lenses, the kinetic energies of the individual particles will remain nearly unchanged during compression. In this case, it is possible, in principle, to apply some "pre-focusing" to the higher energy particles (those nearer to the tail of the beam) to compensate for their weaker focusing in the final lenses. Although kinetic energies of individual particles are not conserved if the beam is not neutralized, one still expects a positive correlation between the particle energies at the beginning of compression and at the end of compression so correction is still assumed to be possible. It is important that the pulse duration is larger upstream than it is at the final focusing lenses. Larger pulse duration makes it easier, from an engineering standpoint, to supply the power needed to 
drive the pulsed correction elements. Nevertheless, it still appears impossible or very costly to provide the needed power for some specific cases that have been studied. In the remainder of this paper we ignore this issue and try to determine if there are other fundamental limitations on how well one might correct. We conclude that there are other important limitations.

In recent years there has been interest in drivers with kinetic energies significantly below $5 \mathrm{GeV}$. For these relatively low kinetic energies some kind of chromatic correction is often essential. Ed Lee has explored time-dependent correction focusing systems to address this issue [2]. The recent study [3] of low energy accelerators for directly driven targets assumes that perfect time-dependent correction is possible.

One relatively obvious way to design a correction system is to place the correction elements upstream of the final focusing elements in such a position that the particle phase advance between correction and final focusing is equal to $n \pi$ where $n \geq 1$ is an integer. In other words, the correction point is an image point of the final lenses. One such system is illustrated in Figure 1. We have simplified this system by representing the final focusing lenses and the correction system by single thin lenses.

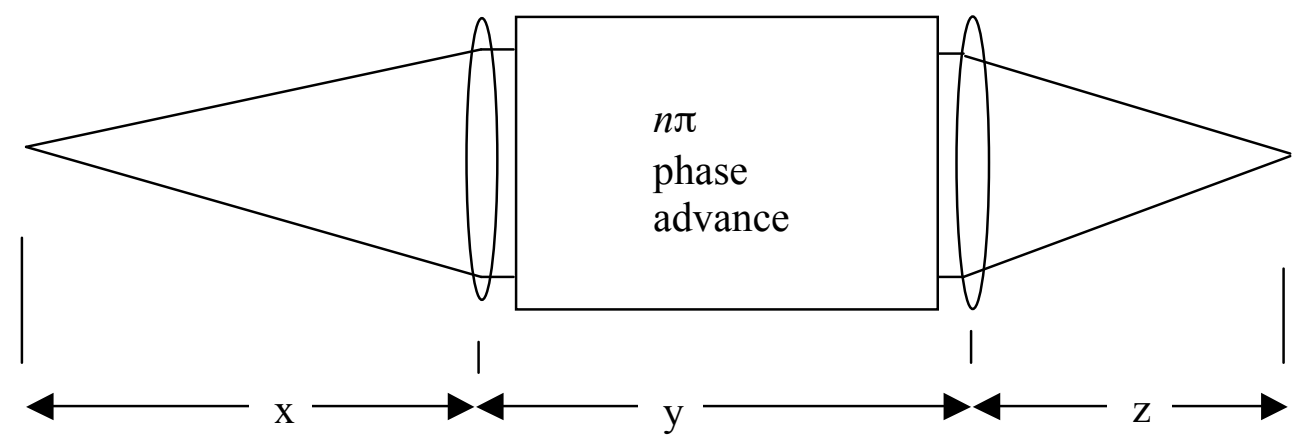

Figure 1. An idealized point-to-point focusing system. The beam starts on the left and drifts a distance $x$ to the first lens. The first lens strength is chosen to produce a parallel beam. The beam then propagates through a section designed to produce a phase advance of $n \pi$ where $n$ is an integer. The length of this section is $y$. The final lens on the right focuses the beam onto the target located a distance $z$ from the lens.

In this system the first (left) lens has a focal length $g$ at the beam energy at the head of the beam. The strength $(1 / g)$ of this lens is increased in time to compensate for the increase in beam energy between the head and tail of the beam. The focal length of the final lens is $f$. Using the usual matrix formalism for optical systems, the transformation matrix for the system is given by

$$
T=\left(\begin{array}{ll}
1 & z \\
0 & 1
\end{array}\right)\left(\begin{array}{cc}
1 & 0 \\
-1 / f & 1
\end{array}\right)\left(\begin{array}{cc} 
\pm 1 & 0 \\
0 & \pm 1
\end{array}\right)\left(\begin{array}{cc}
1 & 0 \\
-1 / g & 1
\end{array}\right)\left(\begin{array}{cc}
1 & x \\
0 & 1
\end{array}\right)
$$


In this equation we have assumed that the section with $n \pi$ phase advance is a harmonically focusing section so that its matrix is plus or minus the identity matrix.

For simplicity we temporarily ignore the distinction between a waist and a focus and assume that we want a point-to-point focus as shown in the figure. The condition for a point-to-point focus is $T_{12}= \pm[x(1-z / f)+z(1-x / g)]=0$. We can achieve this condition by setting $f=z$ and $g=x$ as is clear from the figure. The magnification is given by $T_{11}= \pm(1-z / f-z / g)=\mp z / x$. The beam is typically an order of magnitude larger at the beginning of the system than at the end of the system so $x / z$ in this system would be equal to approximately 10 . In order to be far enough upstream to have a large pulse duration, $y / z$ is also usually of the order of 10 .

Now consider the focusing of a group of particles at the tail of the beam where the mean particle energy is $1+\delta$ times the mean energy at the head of the pulse. Also assume that $\delta$ is much less than 1 . Assuming lens strength is inversely proportional to $1+\delta$, we replace $1 / f$ with $(1-\delta) / f$ and choose a new value of $g$, call it $g^{\prime}$, to retain a point-topoint focus. In practice, the correction would probably be applied by an additional lens at nearly the same position as the original first lens. This strategy would minimize the power and energy required to provide the correction.

Not only does $f$ change but also the $n \pi$-section. In general the matrix for this section is given by

$$
\left(\begin{array}{cc}
\cos k y & \sin k y / k \\
-k \sin k y & \cos k y
\end{array}\right)
$$

where $k y=n \pi$ at the head of the beam. The least change will occur for $n=1$. We set $n=1$ and assume that the wave number $k$ is inversely proportional to momentum. In other words we replace $k$ with $k(1-\delta / 2)$. We can now expand the matrix to first order in $\delta$ obtaining

$$
\left(\begin{array}{cc}
-1 & y \delta / 2 \\
\pi^{2} \delta /(2 y) & -1
\end{array}\right)
$$

Denote the new transformation matrix for the tail of the beam by $T^{\prime}$. The matrix multiplications needed to get the expression for $T^{\prime}$ are somewhat messy and will not be repeated here. The interested reader can easily verify that if one chooses $g^{\prime}$ such that $T_{12}^{\prime}=0$, then the matrix element $T_{11}^{\prime}$ is almost unchanged. Specifically, corrections that are first order in $\delta$ vanish. This result means that both the position of the final focus and the magnification can be nearly preserved and accurate correction is possible. This is confirmed by numerical simulations as will subsequently be shown.

Before showing the results of the simulations, we will discuss some additional issues. One can imagine systems that are different than the system shown in Figure 1. For 
example, one could have a system where the beam radius in the first lens is smaller than the beam radius in the second lens. We did not choose this configuration because chromatic correction is much more important for low-energy beams than for high-energy beams. For low-energy beams it is usually necessary to neutralize the beam. Otherwise, it is difficult to focus and compress the beam because of excessive space-charge forces. On the other hand, it appears difficult to pulse a correcting element in the presence of neutralizing plasma or electrons. We therefore assume that the beam is completely neutralized directly after the correction lens. It is desirable to have a relatively large beam radius in the region where the beam is neutralized. If the beam radius is small, the density of the neutralizing medium must be large. If the required density is large, beam stripping becomes an issue since particles of only one charge state can be adequately focused by the second lens. In some cases instabilities also become an issue. These kinds of considerations lead to a second issue that was first discussed by Ed Lee [4]. We have assumed that $n>0$; however, if $n>0$, there will be intermediate points in the $n \pi$ section where the beam necks down to small radii. Not only is stripping an issue for small beam radii, but also incomplete neutralization. Incomplete neutralization can lead to non-linear forces that degrade beam quality. For these reasons, it seems best to avoid $n>0$, at least for fusion drivers. If $n=0$, the matrix for the $n \pi$-section is no longer diagonal. It is instead given by

$$
\left(\begin{array}{ll}
1 & y \\
0 & 1
\end{array}\right)
$$

The magnification at the head of the beam is unchanged but $T_{11}^{\prime}=-(z+\delta y) / x$ when $g^{\prime}$ is chosen such that $T_{12}^{\prime}=0$. The distance $y$ is often roughly an order of magnitude larger than $z$ so for $\delta \approx 0.1$ the magnification at the end of the beam can be approximately twice as large as at the head of the beam. This result places a constraint on how well one can correct.

Incidentally, if the pulsed lens is assumed to correspond to a set of quadrupoles, one might be able to minimize the required power and energy by using bi-polar pulsing for the correction elements. The middle of the pulse would not be corrected and the beginning and end of the pulse would receive corrections of opposite sign. We have not used bi-polar pulsing, partly because it would require a larger aperture in the final lenses. The larger aperture would be required because the head of the beam would be underfocused leading to some expansion before this part of the beam reaches the final lenses.

We now present numerical simulations of two cases, $n=1$ and $n=0$. Both cases use a standoff from the target of $5 \mathrm{~m}$. The lenses used in these simulations have finite length but are assumed to produce perfect harmonic motion. The strength of the final lens is chosen to be the same as that of a $10 \mathrm{~T}$ solenoid. The kinetic energy and ion mass are the same as those used in reference 3, namely $500 \mathrm{MeV}$ rubidium. Following reference 3, we also assume that the distance between the target and the neutralization point is $60 \mathrm{~m}$. The current upstream of the correction lens is $20 \mathrm{~A}$. The normalized transverse emittance is $1 \mathrm{~mm} \cdot \mathrm{mr}$. The longitudinal emittance is assumed to be $0.04 \mathrm{eV} \cdot \mathrm{s}$; but, since the pulse length at the correction lens is assumed to be $100 \mathrm{~ns}$, the longitudinal emittance has a 
negligible effect on focusing. The length of the final lens is chosen by optimizing the system to produce the smallest focal spot subject to the condition that the beam radius not exceed approximately $8 \mathrm{~cm}$. This condition was imposed to keep the solid angle used by all the beams at a value that might not be impossibly large.

Since the beam is not neutralized until the end of the correction lens, the initial expansion section is chosen to have the appropriate focusing strength and length to bring the beam envelope to zero slope at the appropriate radius. After the system is designed for the head of the pulse, we set $\delta=0.1$ and solve for the value of $g^{\prime}$ that minimizes the mean square radius of the focal spot. The simulations use realistic waist-to-waist focusing rather than the simpler point-to-point focusing used in the analytical calculations.

Figure 2 shows the initial distribution function in transverse $(x-y)$ space. The matched radius is about $1 \mathrm{~cm}$. Reference 3 uses a somewhat larger peak radius in a quadrupole lattice so the value of $1 \mathrm{~cm}$ might correspond to a quadrupole case where the mean radius is $1 \mathrm{~cm}$.

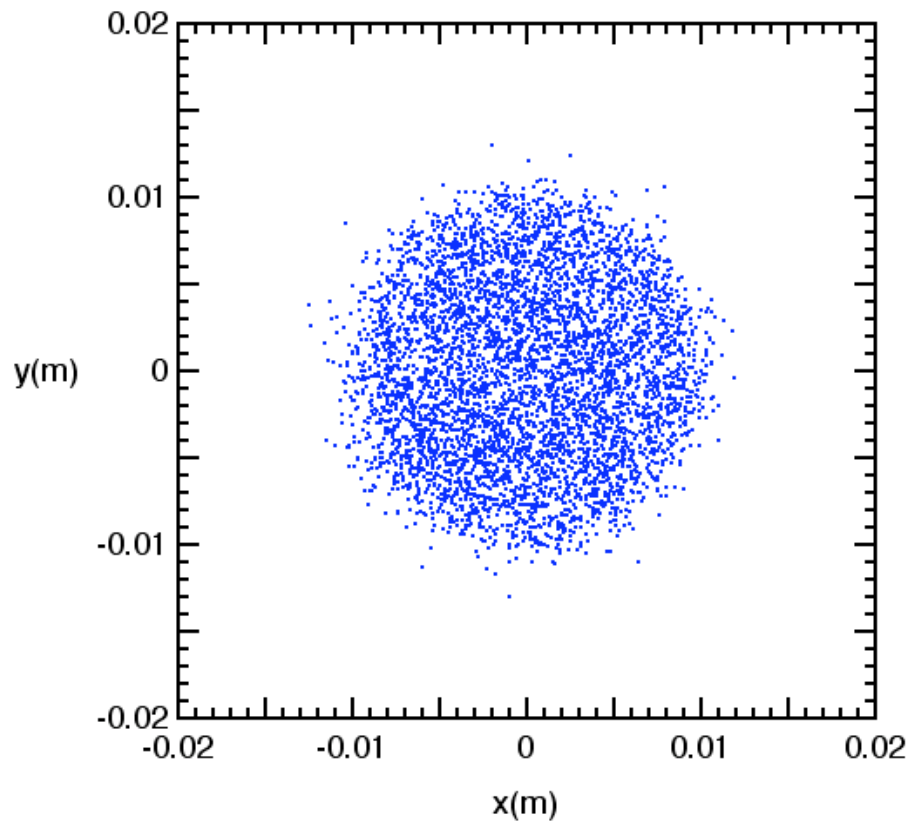

Figure 2. The initial distribution of particles in transverse space. This distribution was obtained by starting the beam with a semi-Gaussian distribution function and then propagating the beam for enough periods to achieve a quasi-equilibrium. To minimize numerical noise, the simulations used 50000 particles but only 5000 are shown. The electric field calculation assumes cylindrical symmetry. 


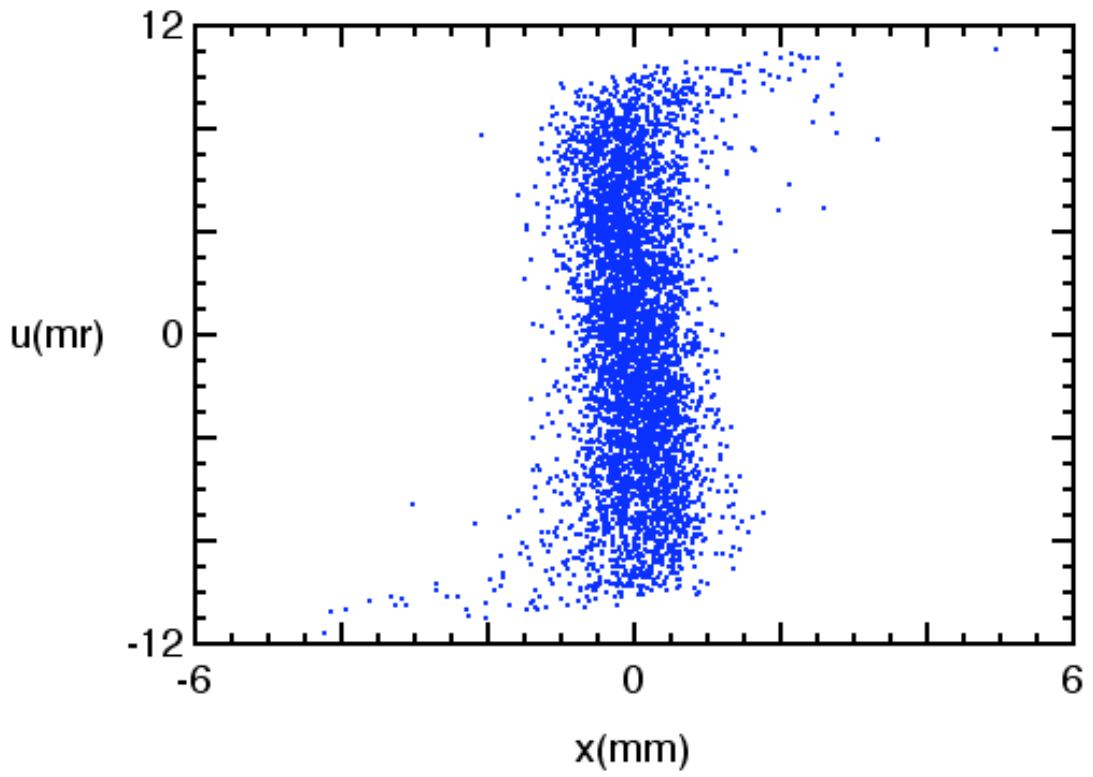

Figure 3. Plot of $x-x^{\prime}$ phase space at the final focus for $n=1$. Note that the nonlinear electric fields that are present before the beam is neutralized distort the phase space, leading to a larger focal spot than would be predicted using simple models with linear fields. For this plot the mean energy of the particles is equal to the energy for which the system is optimized so there is no contribution from beam tilt.

Figure 3 shows the distribution of particles in one transverse direction of phase space (position-angle phase space) at the final focus. This plot corresponds to the case where $n=1$, in other words the case with a perfect section producing a phase advance of 180 degrees. We have also simulated cases using an initial $\mathrm{K}-\mathrm{V}$ distribution function. We are using enough particles that the numerical noise is negligible. The $\mathrm{K}-\mathrm{V}$ distribution gives the expected results and the distortion evident in Figure 3 is not present.

Figure 4 shows the fraction of particles lying within a given radius at the final focus. As explained in the caption, the non-linear fields arising from realistic distribution functions lead to a significant increase in focal spot size. The corresponding plots for the corrected case and for the nominal "on-energy" case with a simple drift between the correction lens and the final lens are nearly identical to Figure 4 so they are not shown. In all these cases, approximately $88 \%$ of the particles lie within a radius of $1 \mathrm{~mm}$. The reason the correction for $n=1$ is essentially perfect is because we ignored stripping and imperfect neutralization. 


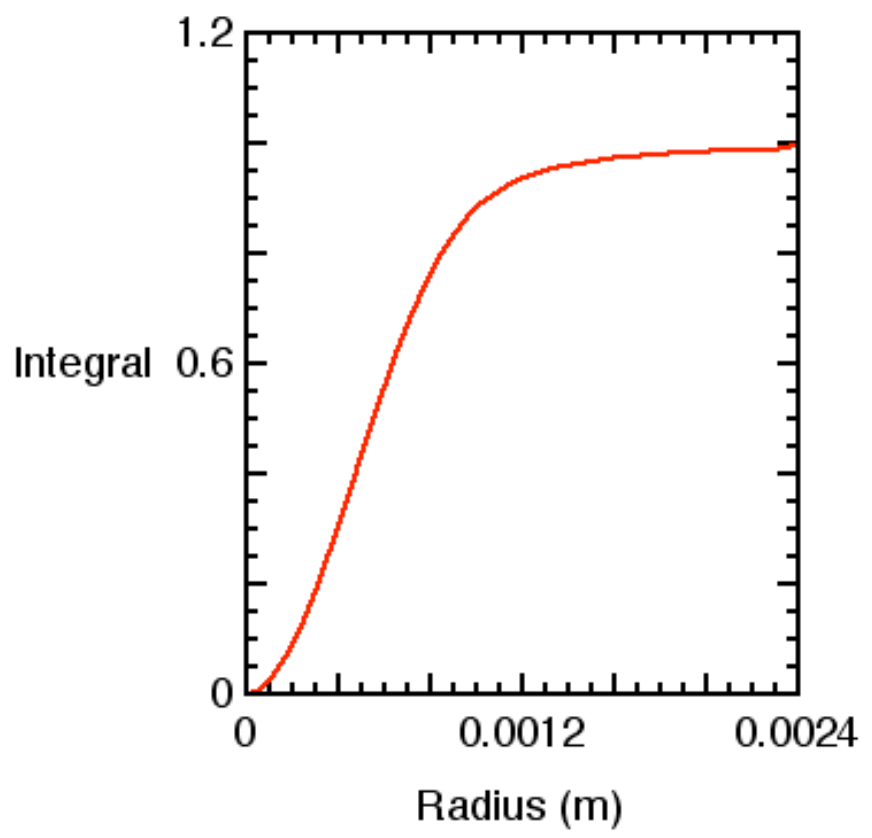

Figure 4. The fraction of particles contained within a given radius for the distribution of Figure 3. Very simple models using a K-V distribution would predict that nearly all the particles would fall within a radius of approximately $0.82 \mathrm{~mm}$. For a semi-Gaussian one might expect about $86 \%$ (the number contained in 2 sigma for a 2-dimensional Gaussian) of the particles to fall within this radius. For this plot $86 \%$ of the particles fall within a radius slightly less than $1 \mathrm{~mm}$ so the effects of the nonlinear electric fields are not negligible.

Figure 5 shows the same type of results as Figure 4. Figure 5 corresponds to the case where the element between the correction lens and the final lens is a simple drift. In this case the radius of the head of the beam remains nearly constant as it drifts. (It is assumed to be perfectly neutralized and the effect of finite emittance is very small because the beam is large.) The energy tilt is assumed to be $10 \%$ corresponding to a velocity tilt of approximately $\pm 2.5 \%$. As noted above, the strength of the correction lens is chosen to give the optimal result. Note that the correction is not perfect -- as we expect from the simple analytic model outlined above. 


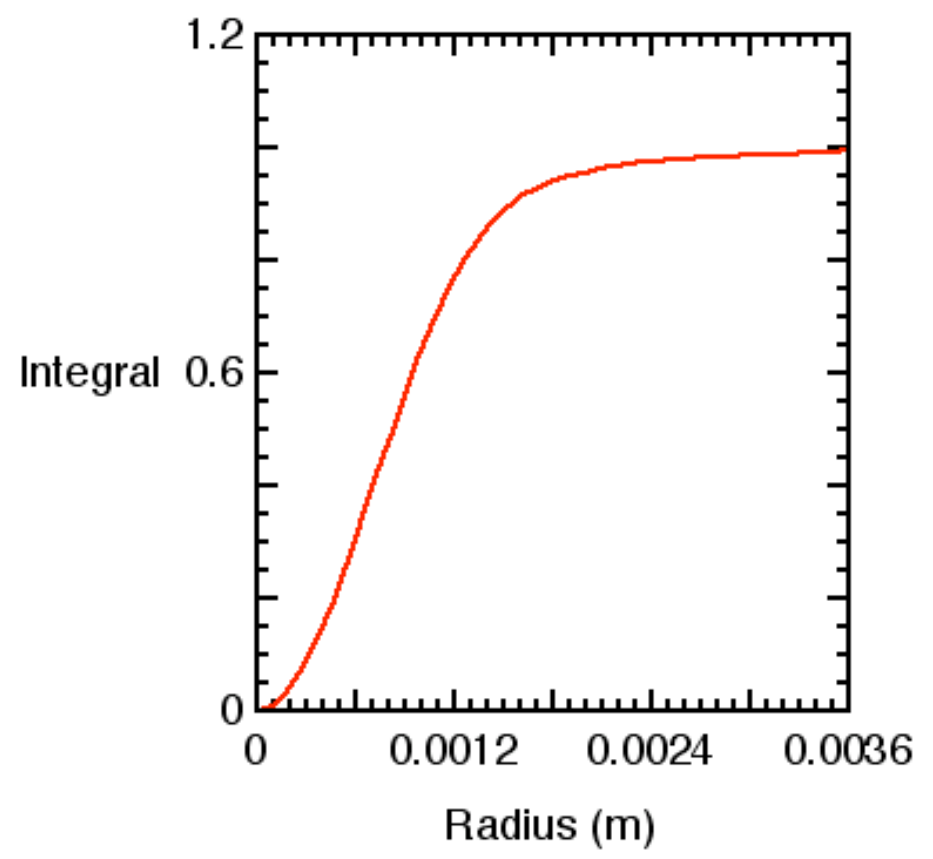

Figure 5. The fraction of particles contained within a given radius for $n=0$ and $\delta=0.1$. The correction lens has been adjusted to minimize the mean square radius of the spot. The cases with no tilt and the $n=1$ case contained approximately $88 \%$ of the particles within a radius of $1 \mathrm{~mm}$. In this case only about $63 \%$ of the particles fall within a radius of $1 \mathrm{~mm}$ so perfect correction is not possible using the example optical system.

There are two important conclusions: (1) It is not possible to obtain nearly perfect correction with the more plausible $n=0$ case. (2) The initial distribution function was assumed to be semi-Gaussian but it was allowed to propagate through enough periods that the spatial distribution approached equilibrium. This equilibrium distribution produces non-linear fields that distort the phase space as shown in Figure 2. Simple formulas such as those used in IBEAM would predict better focusing than we obtain in the simulations. We do, of course, get the same results as the simple formulas if we use a $\mathrm{K}-\mathrm{V}$ distribution function.

It may be possible to design more complex correction systems than our simple example. In particular, it may be possible to use multiple correction elements to minimize both the chromatic aberrations and the changes in magnification. In any case, a lot more work is needed before we can assume, with confidence, that good pulsed correction is possible. In particular the issues of stripping, instability, and imperfect neutralization must be addressed to determine if the $n>0$ option is possible or if other configurations are possible.

The author thanks Peter Seidl, Ed Lee, and Andy Faltens for helpful conversations and suggestions. 


\section{REFERENCES}

1. A. W. Maschke, Comment during discussion following talk by H. Wollnik at the 1984 INS International Symposium on Heavy Ion Accelerators and Their Applications to Inertial Fusion held at the Institute for Nuclear Study, Tokyo, January 23-27, 1984. Also private communication to the author.

2. E.P. Lee, "Time Dependent Focusing", Lawrence Berkeley National Laboratory Report LBNL-59863, (January 2006). Also HIFAN 1455.

3. P. A. Seidl, E.P. Lee, R. O. Bangerter, and A. Faltens, "Induction Linac Driver for a 0.44 MJ Heavy-Ion Direct Drive Target", Lawrence Berkeley National Laboratory Report LBNL-3101E, (February 2010). Also HIFAN 1768.

4. E.P. Lee, "A pulsed electric lens for NDCX", Lawrence Berkeley National Laboratory Report LBNL- ， (July 2007). Also HIFAN 1749. 